\title{
THE INFLUENCE OF THE SURFACE BOUNDARY LAYER ON EVOLUTIONARY MODELS OF JUPITER*
}

\author{
HAROLD C. GRABOSKE, JR. \\ Lawrence Livermore Laboratory, Livermore, Calif., U.S.A.
}

\begin{abstract}
A recent theoretical study of the structure and evolution of Jupiter (Graboske et al., 1974b) is based on a three-stage model of Jovian evolution. The central phase, gravitational contraction of an adiabatic, homogeneous convective fluid system, begins early in solar system evolution and lasts for times of the order of $2 \times 10^{9} \mathrm{yr}$. Good agreement with observed radius and luminosity is achieved for a model with a solar mixture composition. The surface boundary layer has a dominant influence on the evolutionary timescale. Surface boundary factors which are important are the solar energy input, a function of the solar luminosity and the planetary albedo, and the detailed physics of the superadiabatic zone, which depends on the variation of opacity and $\nabla_{\mathbf{a d}}$ with depth. The evolutionary study demonstrates that the current planet cannot be an adiabatic homogeneous fluid throughout. The inclusion of a superadiabatic zone is necessary, and the existence of a heterogeneous (gravitationally layered) fluid interior is possible.
\end{abstract}

\section{Introduction}

The structure and evolution of the planet Jupiter can be investigated by two different approaches. The original method used by DeMarcus (1958) to Hubbard (1970) involves construction of static planetary models utilizing the observed mass and radius. These static models have been successful in allowing estimation of chemical composition and semi-quantitative determinations of interior densities and temperatures. The second approach, recently carried out by Graboske, Pollack, Grossman and Olness (1974b), hereinafter referred to as GPGO), uses a stellar structure method to calculate an evolving model of the planet, specifying only the mass and chemical composition. This evolutionary method has produced models of the planet which not only agree well with observational characteristics such as mass, radius and luminosity, but give us significant information on the energetics and interior conditions of the Jovian system.

\section{Construction of Evolutionary Model Sequences}

The evolutionary study deals with the central phase of a three stage process. The first phase, the assembly stage, postulates either a stellar gaseous collapse origin or a cold accretion origin, this protoplanetary phase culminating in a stable quasistatic configuration at the top of the Hayashi track. The central phase is the fluid contraction state, gravitational contraction along the Hayashi track for times exceeding $10^{9} \mathrm{yr}$. A third phase is suggested, the post-fluid contraction stage, where additional energy sources become important, adding to the energy released from stored heat of prior contraction and from current contraction. These additional sources may be energy released by phase changes (Smoluchowski, 1967) or by gravitational separation (Salpeter, 1973;

* Work performed under the auspices of the U.S. Atomic Energy Commission. 
Smoluchowski, 1973). The evolutionary study of GPGO is concerned only with the fluid contraction stage.

The basic assumptions are that the planetary model is spherical nonrotating, nonmagnetic, and is composed of a solar mixture composition $(X=0.74, Z=0.02)$. The fundamental structure chosen is that originally proposed by Hubbard, an adiabatic, homogeneous (fully mixed) fluid mixture. The calculation is started from an extended gaseous condition $\left(R=15.6 R_{\mathrm{J}}\right)$ a stable, convective configuration high on the Jovian Hayashi track. It is evolved to a time approaching $10^{10} \mathrm{yr}$, using a Henyey-type star code, which solves the stellar structure equations for a convective system. The surface boundary condition is established from planetary model atmospheres calculated with the method of Pollack and Ohring (1973). These flux corrected atmospheres cover a temperature range from $50 \mathrm{~K}$ to $2000 \mathrm{~K}$, including as principal opacity sources hydrogen, ammonia, water vapor and methane. An additional feature is the inclusion of a solar energy component, characterized as $T_{\odot}$ an effective insolation temperature, which is a function of the solar luminosity and the planetary orbit and albedo. The thermodynamic properties for the solar mixture are taken from Graboske et al. (1974a), where fully mixed fluids composed of a solar mixture $(X=0.74, Z=0.02)$ were calculated by two theoretical approaches. The high density (metallic) regime is studied using a version of Thomas-Fermi theory developed for mixtures and strongly coupled coulomb systems. The low density (molecular, atomic and plasma) regime is studied using a free energy minimization model, which incorporates recent high pressure experimental results for the molecular fluid, as well as Monte Carlo results for the dense plasma.

The results of the study are evolutionary models for a Jovian mass object that have two different phases. The early evolution produces a stellar object, behaving in a manner similar to the low mass premain sequence stars $\left(0.01 \leqslant M / M_{\odot} \leqslant 0.20\right)$, with relatively high effective temperatures $(1600 \mathrm{~K})$, luminosities $\left(\log L / L_{\odot}=-2.3\right.$ initially), and internal temperatures $\left(T_{c \max }=40600 \mathrm{~K}\right.$ at $\left.4.1 \times 10^{4} \mathrm{yr}\right)$. This phase is succeeded by a degenerate dwarf cooling phase, during which the model contracts, cools and approaches very close to the observed radius and luminosity of the present Jupiter $\left(R / R_{\mathrm{J}}=0.98, L / L_{\mathrm{J}}=1.00\right)$. A number of parametric studies were made, computing evolutionary sequences while varying quantities whose values have a range of uncertainty, but the best agreement is achieved for the standard constitutive physics and the $(X=$ $=0.74, Z=0.02$ ) solar mix composition. A significant result was found, obtainable only from an evolutionary calculation, concerning the duration of the fluid contraction stage. The age of the model which agrees best with the current $R_{\mathrm{J}}, L_{\mathrm{J}}$ values is only $1.87 \times 10^{9} \mathrm{yr}$. The sensitivity of this relatively short lifetime to various parameters such as chemical composition and $T_{\odot}$ were investigated, and the results are instructive.

\section{Effects of the Surface Boundary Layer}

The construction of evolutionary sequences for different constitutive physics resulted in a range of model tracks in the $\log L, \log T_{e}$ plane. The location of the $4.5 \times 10^{9} \mathrm{yr}$ 
models are shown in Figure 1. The solar mix standard model $\left(T_{\odot}=89 \mathrm{~K}\right)$, track passes very close to the observational values for Jupiter $(\oplus)$ at $1.87 \times 10^{9} \mathrm{yr}$, but is over 3 times less luminous at $4.5 \times 10^{9} \mathrm{yr}$. The other models are the same epoch models for different values of $T_{\odot}(3 \mathrm{~K}, 108 \mathrm{~K})$ representing the extremes of the solar energy deposition, for different chemical composition (pure hydrogen), for a modified set of

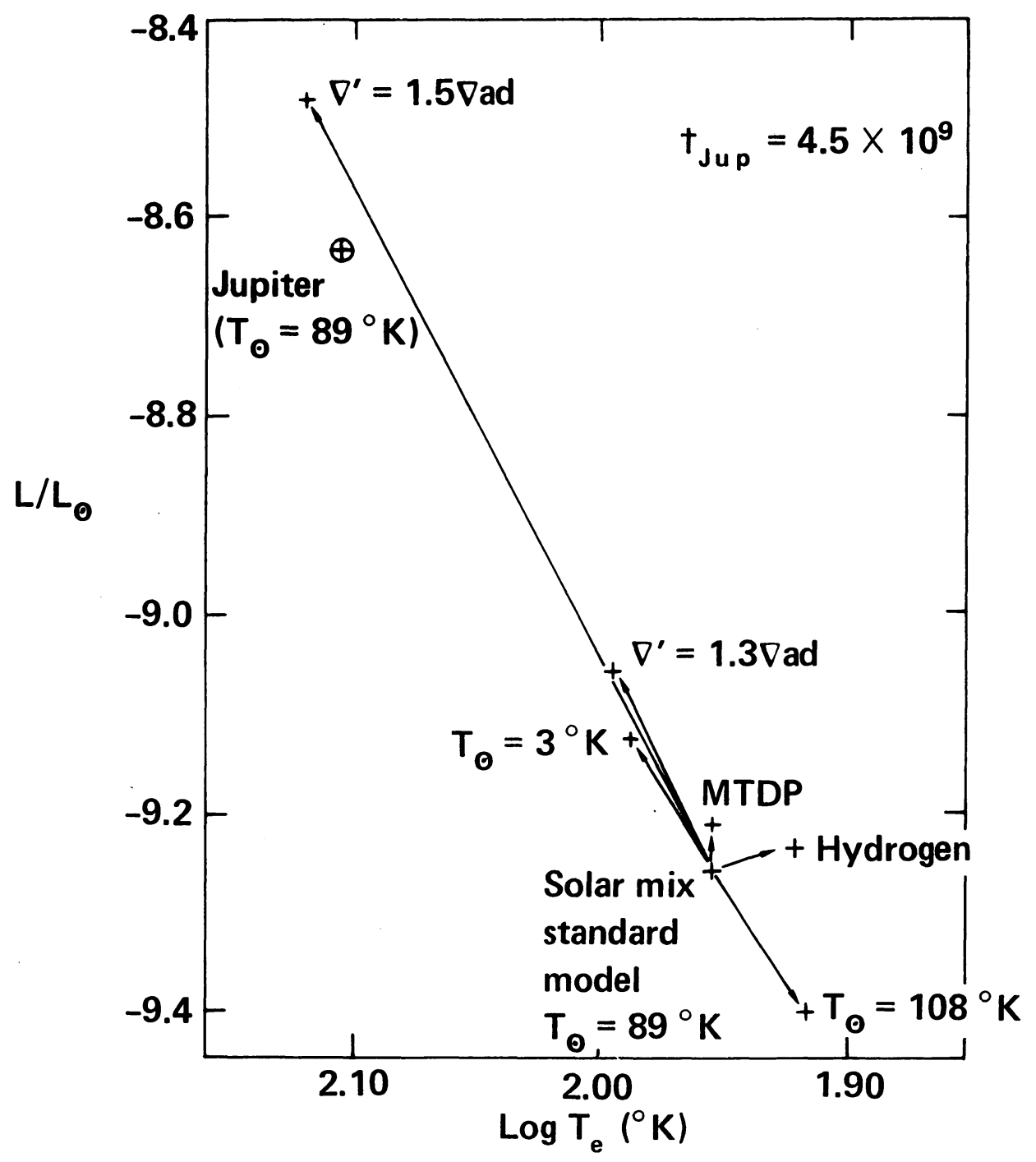

Fig. 1. Planetary models in the $\log L-\log T_{e}$ plane at $t-4.5 \times 10^{9}$ years. The observed values for Jupiter $\left(\oplus\right.$ ) lie above the standard model (solar mix, $T_{\odot}=89 \mathrm{~K}$ ). The positions of the models at $4.5 \times 10^{9} \mathrm{yr}$ for different $T_{\odot}$ values, different chemical composition (pure hydrogen), modified constitutive physics (MTDP), and arbitrary superadiabatic zones $\left(\nabla^{\prime}\right)$ are indicated. 
thermodynamic properties (MTDP), for a modified set of model atmospheres (change too small to be discriminated from the standard model) and for an artificial superadiabatic zone of varying extent $\left(\nabla^{\prime}\right)$. The primary conclusion is that no change in the model atmospheres, thermodynamics, chemical composition or solar energy component can extend the lifetime of the fluid contraction stage enough to yield an adiabatic homogeneous fluid model which has $R_{\mathrm{J}}, L_{\mathrm{J}}$ and a lifetime $\left(t_{\mathrm{J}}\right)$ comparable to that of Jupiter.

The surface boundary layer effects, $T_{\odot}$ and $\nabla^{\prime}$, are presented in more detail in Figures 2 and 3. In Figure 2, the effective age of the model at which its luminosity equals that of the present Jupiter $\left(t_{\mathrm{J}}^{L_{J}}\right)$ is given as a function of $T_{\odot}$ and $\nabla^{\prime}$. The $3 \mathrm{~K}$ value of $T_{\odot}$ represents an absolute minimum, a $0.00095 M_{\odot}$ object evolving without a stellar companion, while the $89 \mathrm{~K}$ and $108 \mathrm{~K}$ values represent the maximum and minimum planetary albedo values. A temperature of $102 \mathrm{~K}$ would be the most probable value of $T_{\odot}$. Variations in $t_{\mathbf{J}}^{L_{\mathbf{J}}}$ of approximately $10 \%$ are achieved, and hence no reasonable variations in $T_{\odot}$, due to secular changes in the solar luminosity or changing albedo of the contracting cooling planet are capable of increasing the lifetime significantly.

The other surface boundary layer effect is more difficult to assess, and it is only done in an arbitrary and approximate manner in GPGO. Since an accurate treatment of a stellar model containing a superadiabatic zone requires use of the mixing length theory incorporating both opacity and $\nabla_{\mathrm{ad}}$ as a function of depth, a simple approximation was used. The superadiabatic gradient $\nabla^{\prime}$ was set equal to a multiple of the adiabatic

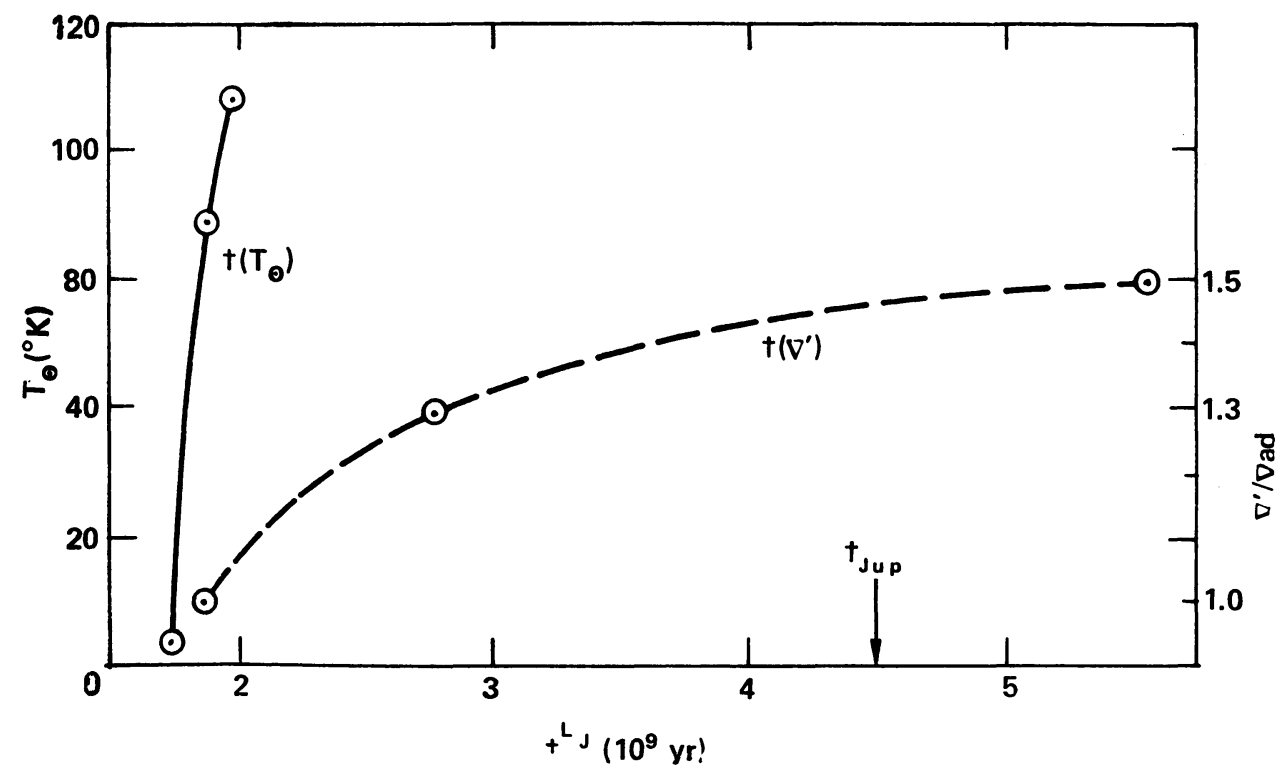

Fig. 2. The dependence of model age at $L=L_{J}$ as a function of insolation temperature and of superadiabatic gradient. 

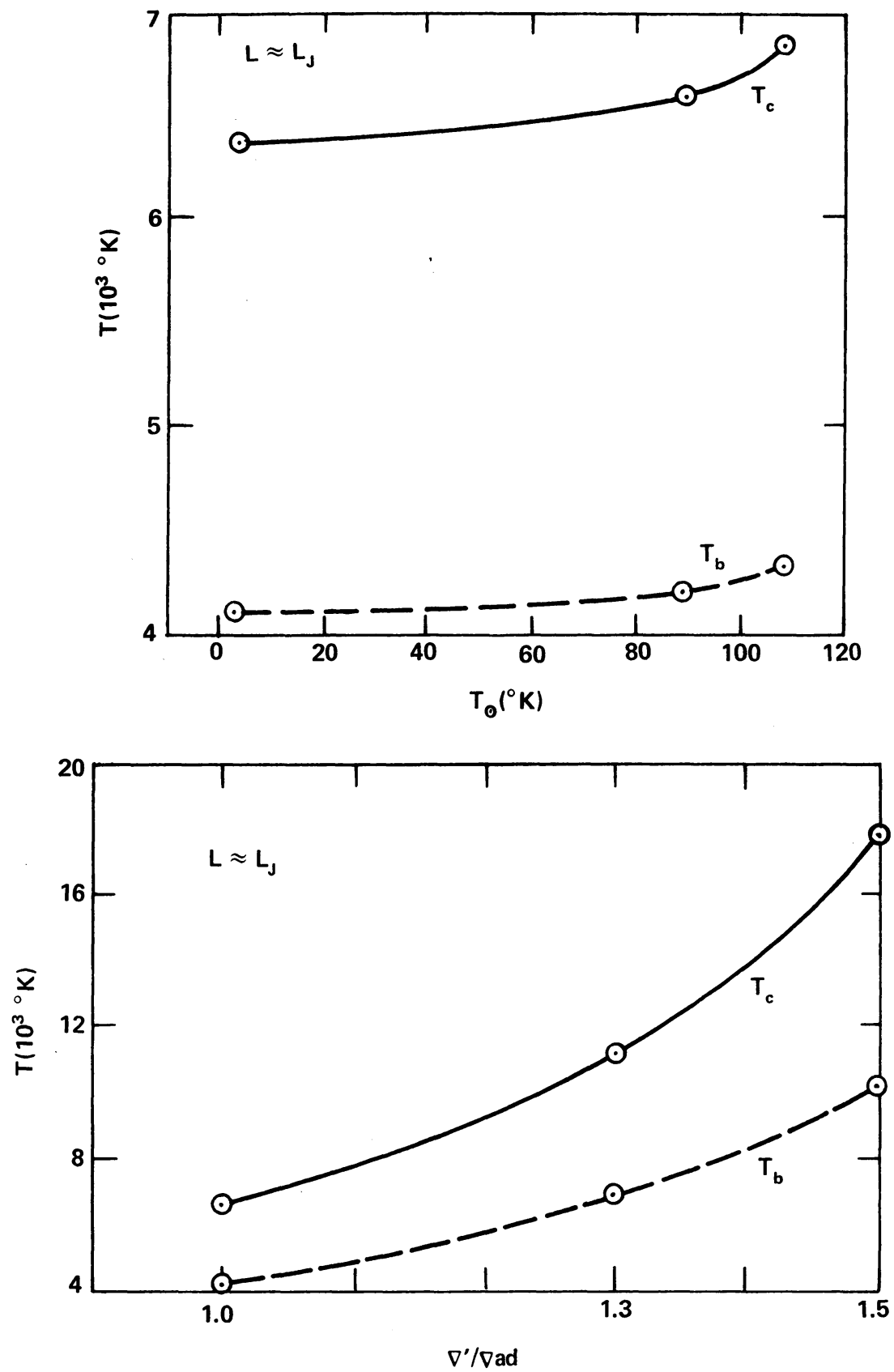

Fig. 3. The dependence of model central temperature $\left(T_{c}\right)$ and core boundary temperature $\left(T_{b}\right.$, where $M / M_{\mathrm{J}}=0.97$ ) are given as a function of $T_{\odot}$ and $\nabla^{\prime}$, for the track location $L=L_{\mathrm{J}}$. 
gradient over a mass range $\Delta M$ in the outer envelope of the model. The size $(\Delta M)$ and strength $\left(\nabla^{\prime} / \nabla_{\text {ad }}\right)$ of the artificial superadiabatic zone were chosen by comparison with the surface layers of low mass premain sequence stars $\left(0.01 M_{\odot}-0.10 M_{\odot}\right)$, with $\Delta M=10^{-4} M_{\text {total }}$. Three values of $\nabla^{\prime} / \nabla_{\text {ad }}$ were chosen, 1.0 (the standard, adiabatic model), 1.3 and 1.5. The dependence of $t_{\mathrm{J}}^{L_{\mathrm{J}}}$ on $\nabla^{\prime}$ is shown in Figure 2 to be very strong, by far the dominant influence on the planetary structure. As determined from Figures 1 and 2 , a value of $\nabla^{\prime} / \nabla_{\mathrm{ad}} \approx 1.45$ would yield an adiabatic homogeneous fluid model with $R, L$ and $t$ all near the Jovian values. The track would lie slightly to the right of the planetary values, indicating that possibly a slightly higher He content is necessary.

In addition to modifying the observational characteristics of the models, the $T_{\odot}$ and $\nabla^{\prime}$ parameters also modify the internal structure. The modifications in internal temperature are shown in Figure 3 , for models with $L \approx L_{\mathrm{J}}$. These models have widely differing ages, since $L=L_{\mathrm{J}}$ at very different times for various values of $T_{\odot}$ and $\nabla^{\prime}$. The central temperatures $\left(T_{c}\right)$ for the $T_{\odot}$ variation range from $6368 \mathrm{~K}$ to $6842 \mathrm{~K}$, while the temperatures at the core boundary $\left(T_{b}\right)$, where $M / M_{\mathrm{J}}=0.97$, range from $4072 \mathrm{~K}$ to $4339 \mathrm{~K}$. As was the case for the lifetime variation, the influence of $\nabla^{\prime}$ is far stronger than that of $T_{\odot}$. The central temperature as a function of $\nabla^{\prime}$ increases from $6600 \mathrm{~K}$ (adiabatic) to $16893 \mathrm{~K}$, while the temperature at the core boundary increases from $4204 \mathrm{~K}$ to $10018 \mathrm{~K}$. The direction and relation between the lifetime and internal temperature changes are obviously correlated. The stronger the superadiabatic zone, the slower the energy release from the interior, so that the increasing lifetime with increasing $\nabla^{\prime}$ is accompanied by higher thermal content of the core.

The construction of evolutionary model sequences incorporating a realistic superadiabatic zone, using mixing length theory with appropriate outer envelope opacities and thermodynamic properties for the dissociating, ionizing gases, is currently being carried out. The results of this further step in the evolutionary study should provide a definitive model sequence for the fluid contraction stage.

\section{Evolutionary Structure Subsequent to the Fluid Contraction Stage}

The inclusion of a realistic superadiabatic zone will give a specific age for the $L=L_{\mathrm{J}}$ model, within the limits allowed by the uncertainties in $T_{\odot}$, chemical composition and the constitutive physics, all of which are seen to have fairly small effects. If $t_{\mathbf{J}}^{L_{\mathbf{J}}}$ is $4.5 \times 10^{9} \mathrm{yr}$ or greater, the superadiabatic, homogeneous fluid model can be used to describe the structure of present Jupiter. The size of the effective $\nabla^{\prime}$ required to yield such lifetimes $\left(\nabla^{\prime} / \nabla_{\mathrm{ad}} \approx 1.45\right)$ will produce a dense $\left(4 \mathrm{~g} \mathrm{~cm}^{-3}\right)$ and hot $\left(T_{c} \approx 16000 \mathrm{~K}\right.$, $T_{b} \approx 9500 \mathrm{~K}$ ) interior, which would correlate well with a model assuming ionized (metallic) fully mixed components. However, if the superadiabatic model sequence has $t_{\mathrm{J}}^{L_{\mathrm{J}}}<4.5 \times 10^{9} \mathrm{yr}$, then an additional source of energy must be sought to achieve agreement with the observed planet.

Two such sources have been proposed, by Smoluchowski and Salpeter. Smoluchowski (1967) suggested that as the planetary interior cooled, hydrogen would solidify, 
the fluid helium would diffuse out of the hydrogen solid under the influence of the gravitational field and a multilayer structure would result. Two additional sources of energy are available in this model: the latent heat of fusion released by the freezing hydrogen plus the gravitational energy released by the inward-diffusing helium. Salpeter (1973) proposed a different mechanism for gravitational layering, pointing out that a neutral helium fluid might be able to diffuse out of a metallic hydrogen fluid against a convective mixing flow. This immiscible fluid model would produce a differentiated model with consequent gravitational energy release. As recently estimated by Smoluchowski (1973), gravitational layering can produce the observed Jovian luminosity from the separation of a small fraction of the Jovian mass $(1 \%)$.

The accurate evolutionary model of the superadiabatic homogeneous fluid will give quantitative values for the energy produced by current gravitational contraction and that produced by release of heat stored from prior contraction. These two sources contribute approximately equivalent amounts for the time period $t>10^{9} \mathrm{yr}$. This will in turn allow an accurate estimation of the energy derived from gravitational differentiation, and provide information on the size of the planetary mass involved in the layering process. A second advantage of obtaining an accurate homogeneous fluid sequence is that, in combination with recent advances in the theory of dense fluids and fluid mixtures, it will allow a determination of possible phase transitions occurring in the planet. The molecular fluid-metallic fluid phase transition has been accepted since DeMarcus' study (1958). The possibility of a metallic solid phase, at least for timescales of $10^{10}$ yr or less is almost certainly ruled out by the high temperatures existing in the central zone of the planet. Using a recent study of the dense hydrogen fluid region by Ross (1973), an analysis of the pure hydrogen model sequence (adiabatic, homogeneous) indicated that a thin shell of solid molecular hydrogen would form at $R / R_{\mathrm{J}} \approx 0.6$ and slowly grow outward, at $t=2.0 \times 10^{9} \mathrm{yr}$. The inclusion of even a modest superadiabatic zone in the pure hydrogen model would raise the internal temperatures sufficiently so that no solid molecular hydrogen could form anywhere in the interior. The appearance of solid phase in a hydrogen-helium mixture is more difficult to study, but it is unlikely to be stable at significantly higher temperatures than the hydrogen solid. Thus, the possibility of solid phases in a Jovian model with $t \lesssim 4.5 \times 10^{9} \mathrm{yr}$ is improbable, and if gravitational layering is found to be required by planetary energetics, Salpeter's immiscible fluid is the favored mechanism.

\section{References}

DeMarcus, W.: 1958, Astron. J. 63, 2.

Graboske, H. C., Jr., Olness, R. J. and Grossman, A. S.: 1974a, Astrophys. J., to be published.

Graboske, H. C., Jr., Pollack, J. B., Grossman, A. S., and Olness, R. J.: 1974b, Astrophys. J., to be published.

Hubbard, W. B.: 1970, Astrophys. J. 162, 687.

Pollack, J. B. and Ohring, G.: 1973, Icarus 19, 34.

Ross, M.: 1973, J. Chem. Phys., to be published.

Salpeter, E. E.: 1973, Astrophys. J. 181, L83.

Smoluchowski, R.: 1967, Nature 215, 691.

Smoluchowski, R.: 1973, Astrophys. J. 185, L95. 


\section{DISCUSSION}

Trafton: Do experiments involving such transient phenomena really give the equilibrium properties of hydrogen?

Graboske: Yes. The time resolution, for example, is some 50 times that required to determine the transient behaviour.

Williams: With a graduate student, R. Donnison, I attempted a similar calculation and similar problems to what you mentioned occurred, namely the radius being too short. The problems you outlined are therefore real I think.

Graboske: As shown by DeMarcus, cold hydrogen models are not capable of reproducing the observed radius of either giant planet, and these more recent studies show that adiabatic models with hot interiors are also too large. In addition to the surface boundary layer effects mentioned, our study of the evolutionary sequences included extensive analyses of the possible effects of changes in the thermodynamic properties, in the model atmospheres and in chemical composition. Changes in lifetime at $L=L_{\mathrm{J}}$ are typically no greater than 20 to $30 \%$ for any of these variations, with the exception of the inclusion of the superadiabatic zone.

Hide: The presence of a thin 'crust' of high viscosity would greatly simplify the problem of interpreting the variable rotation of Jupiter (as evinced by observations of the radio-period and atmospheric features). It will be important in your future calcultions to set a crust on the basis of various theoretical models and not simply to consider completely fluid models.

Graboske: This is an important point I would like to respond in three parts. (1) The structure method is not limited to fluid models. The fluid model was adopted for simplicity and because the recent models of Hubbard and Trubitsyn indicated high internal temperatures incomptatible with the presence of solid material. In future studies we intend to allow for the formation of solid zones in the interior if the physical conditions require them.

(2) The liquid-solid high pressure phase diagram of $\mathrm{H}-\mathrm{He}$ is totally unknown at present, and that of pure hydrogen is only partially complete. Using the hydrogen phase diagram of M. Ross of the Lawrence Livermore Laboratory, I have observed that conditions favouring the appearance of a thin shell of solid molecular hydrogen occur in the pure hydrogen model sequence. This shell would form at $0.6 R_{\mathrm{J}}$, at about $2.5 \times 10^{9} \mathrm{yr}$, and slowly grow outward. However, this is an adiabatic fluid model; inclusion of a superadiabatic zone will increase the internal temperatures in the sense shown above for the solar mixture models. This would delay or completely prevent the appearance of a solid layer. Its possible formation in a superadiabatic, $\mathrm{H}-\mathrm{He}$ model is even less probable.

(3) Even if final evolutionary models yield a fluid planetary structure, the presence of a thin crust in the outermost layers might be permitted. It is possible it could be inserted without significantly perturbing the first model, although its calculation would require special procedures. 